\title{
Intraocular adrenaline maintains mydriasis during
}

\section{cataract surgery}

\author{
Melanie C Corbett, Andrew B Richards
}

\begin{abstract}
Cataract surgery is performed more easily if mydriasis can be maintained until the intraocular lens has been inserted. Intraocular irrigation with adrenaline is thought to be of benefit in this respect, and is used by some surgeons but not others. This prospective double blind controlled trial assessed the efficacy and safety of using perioperative adrenaline during extracapsular cataract surgery, as an adjunct to preoperative topical mydriatics. Seventy patients were randomised to receive intraocular irrigation fluid with or without 1:1000 000 adrenaline. The adrenaline entering the eye through the anterior capsulotomy needle helped to resist the miosis induced by expression of the nucleus $(7 \cdot 1$ versus $6.5 \mathrm{~mm}$ ). The mydriasis maintained during irrigation aspiration was significantly greater in the group receiving adrenaline (6.6 versus $6.0 \mathrm{~mm}, p<0.02)$. Their pupil diameters were also significantly larger at 20 minutes $(\mathbf{p}<0.001)$ and 30 minutes $(\mathbf{p}<0.01)$ into surgery. Pupillary constriction to a diameter of less than $5 \mathrm{~mm}$ occurred more frequently in the group not receiving adrenaline. Pulse rate and blood pressure in the 27 patients who had local anaesthesia showed no significant difference between the treatment groups $(p>0.05)$, and there was no significant variation from baseline $(p>0.05)$. Intraocular irrigation with adrenaline 1:1 000000 is a safe and effective means of maintaining mydriasis during cataract surgery. (Br F Ophthalmol 1994; 78: 95-98)
\end{abstract}

Cataract extraction is performed more easily if mydriasis can be maintained until the intraocular lens has been inserted. ${ }^{1}$ Failure to maintain mydriasis during surgery may increase the risk of damage to the iris, incomplete clearance of soft lens matter, or more importantly, rupture of the posterior capsule. ${ }^{2}$

The size of the pupil is determined by the net effect of opposing forces: adrenergic sympathetic activity stimulating the dilator pupillae thereby inducing mydriasis ${ }^{3}$; and cholinergic parasympathetic activity acting on the sphincter pupillae with the production of miosis. Adrenaline directly stimulates the dilator pupillae, but when applied to the conjunctiva, the 1:1000 solution does not penetrate into the normal eye in sufficient quantity to have an obvious mydriatic effect. ${ }^{4}$

Preoperative mydriasis for cataract extraction is usually achieved by a topical adrenergic agonist with good penetration of the cornea, such as phenylephrine $10 \%$, in combination with an anticholinergic drug such as cyclopentolate $1 \%$. During surgery there is a tendency for the pupil to constrict, particularly following manipulation of the iris. ${ }^{5}$ Once the anterior segment has been opened, adrenaline solution can be infused directly into the anterior chamber to stimulate the dilator pupillae. Many surgeons use adrenaline in the intraocular infusion during cataract surgery, but there appears to have been no controlled study so far to confirm that it has a beneficial effect on mydriasis beyond that produced by the preoperative drops.

\section{Patients and methods}

Seventy patients ( 33 men, 37 women; mean age 75 years) undergoing routine extracapsular cataract extraction were randomly allocated to receive intraocular irrigation with or without adrenaline 1:1000000. All patients underwent full ophthalmic and general medical history and examination, including measurement of the pulse rate and blood pressure, and an electrocardiogram; and all gave informed consent before inclusion in the trial. Exclusion factors were a history of: (a) allergy to any of the drug components to be used; (b) topical or systemic therapy capable of interfering with pupil diameter; (c) previous intraocular inflammation.

All patients received four drops of both cyclopentolate $1 \%$ and phenylephrine $10 \%$ during the 1 hour preceding surgery. No patients received sympathomimetic agents with the local anaesthetic injection. An average of $40 \mathrm{ml}$ balanced salt solution was used for intraocular irrigation during surgery. Irrigation fluid first entered the eye through the anterior capsulotomy needle, when the rate of flow was slow. After expression of the nucleus, a faster flow was used during irrigation aspiration of the soft lens matter.

Investigators, with no knowledge of the type of irrigation fluid, measured the pupil diameters before instillation of mydriatic drops, before anaesthesia, and before surgery, then at 10,20, and 30 minutes during surgery. Measurements were also taken in relation to specific surgical events: before and after expression of the nucleus, and after irrigation aspiration of the soft lens matter. Preoperative measurements were made by comparison with standard black circles of known diameter. Intraoperative measurements were made by calipers viewed through the operating microscope.

The average effect of the two treatment regimens was assessed by calculating the mean pupil diameters at each time, and comparison was made using an unpaired Student's $t$ test. It is also clinically important to consider the reliability of each treatment in producing a minimum desired effect. Before starting the study, it was decided on the clinical judgment of several experienced surgeons, that a minimum pupil diameter of 
$5 \mathrm{~mm}$ is required for miosis not to significantly increase the difficulty of the procedure. Maintenance of a pupil diameter of more than $5 \mathrm{~mm}$ was one of the criteria used for the analysis of the additional benefit given by adrenaline in the intraocular irrigation fluid, and comparison between the two treatment groups was made using a $\chi^{2}$ test.

Cardiovascular indices were recorded for only the 32 patients under local anaesthetic, to avoid the production or masking of changes by general anaesthesia. Of these, 12 patients received irrigation containing adrenaline, and 20 received no adrenaline. The pulse rate and blood pressure were recorded using an automatic cuff, at the same time intervals as the pupil diameter. Comparison between the two groups was made using an unpaired Student's $t$ test. Continuous electrocardiographic monitoring was performed throughout surgery.

Adverse local or systemic events were recorded. An unpaired Student's $t$ test was used to analyse the difference between the two groups for pupil diameter, pulse rate, and systolic and diastolic blood pressure.

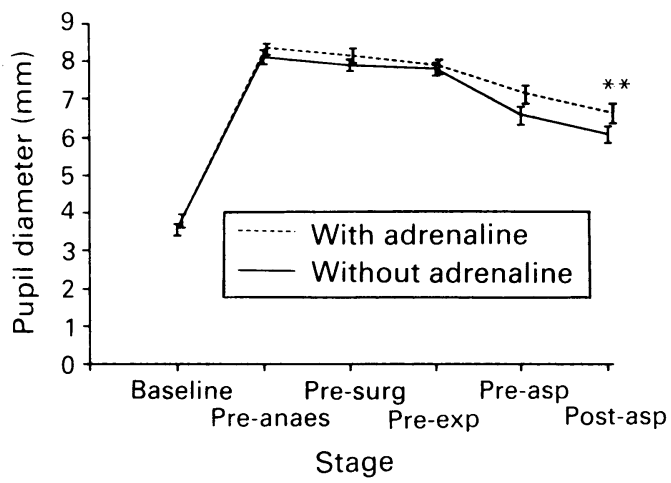

Figure 1 Mean pupil diameters recorded according to stage of surgery both with and without adrenaline in the intraocula infusion. Measurements were made before administration of drops (baseline), before anaesthesia (pre-anaes), and before surgery (pre-surg), then before expression of the nucleus (preexp), and before (pre-asp) and after (post-asp) aspiration of the soft lens matter. Data points are displaced slightly in time to facilitate comparison of standard error bars (significance of the difference: ${ }^{\star \star} p<0.02$, no stars $p>0.05$ ).

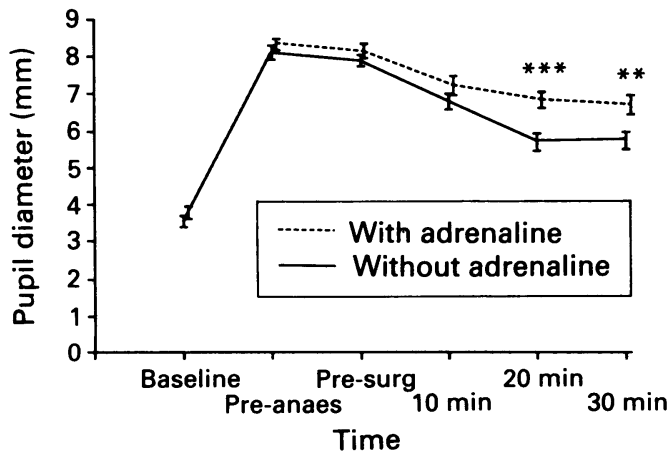

Figure 2 Mean pupil diameters for surgery with and without adrenaline in the intraocular infusion. Measurements were made before administration of drops (baseline), before anaesthesia (pre-anaes), and before surgery (pre-surg), then at 10,20, and 30 minutes during surgery. Data points are displaced slightly in time to facilitate comparison of standard error bars (significance of the difference: $\star \star \star p<0.001$, $\star \star p<0.01$, no stars $p>0.05$ ).
Table 1 Percentage of patients with pupil diameters of less than $5 \mathrm{~mm}$ at various times and stages during surgery (actual numbers of patients in brackets). Pupil constriction to a diameter of less than $5 \mathrm{~mm}$ occurred more frequently in the group not receiving adrenaline $(p<0.001)$

\begin{tabular}{lll}
\hline Time or stage of surgery & $\begin{array}{l}\text { Without } \\
\text { adrenaline }\end{array}$ & $\begin{array}{l}\text { With } \\
\text { adrenaline }\end{array}$ \\
\hline Total number of patients & 43 & 27 \\
Before expression & $0 \%$ & $0 \%$ \\
Before aspiration & $16 \%(7)$ & $0 \%$ \\
After aspiration & $21 \%(9)$ & $7 \%(2)$ \\
$10 \mathrm{~min}$ & $9 \%(4)$ & $4 \%(1)$ \\
$20 \mathrm{~min}$ & $21 \%(9)$ & $4 \%(1)$ \\
$30 \mathrm{~min}$ & $21 \%(9)$ & $9 \%(3)$ \\
\hline
\end{tabular}

\section{Results}

The pupil diameters of the two groups were similar at the start of surgery and before expression of the nucleus $(7 \cdot 8 \mathrm{~mm}$ mean pupil diameter in both groups). The adrenaline entering the eye through the anterior capsulotomy needle helped to resist the miosis induced by expression of the nucleus $(7.1$ versus $6.5 \mathrm{~mm})$. The mydriasis maintained during irrigation aspiration was significantly greater in the group receiving adrenaline $(6.6$ versus $6.0 \mathrm{~mm}, \mathrm{p}<0.02)$ (Fig 1 ). Their pupil diameters were also significantly larger at 20 minutes $(\mathrm{p}<0.001)$ and 30 minutes $(\mathrm{p}<0.01)$ into surgery (Fig 2). Pupil constriction to a diameter of less than $5 \mathrm{~mm}$ occurred more frequently in the group not receiving adrenaline $(p<0.001)$ (Table 1). There was one case of posterior capsule rupture, which occurred in a

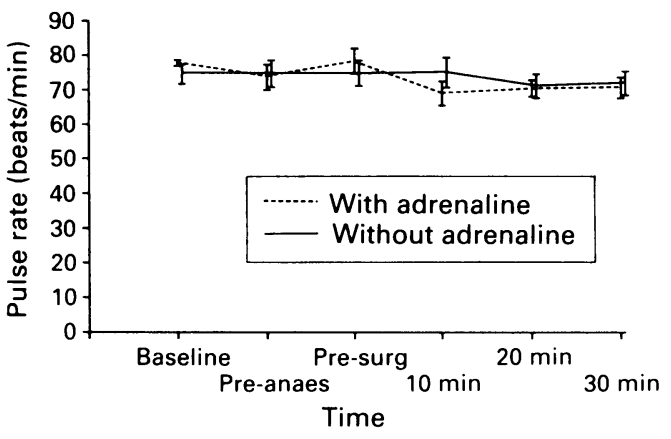

Figure 3 Pulse rate recorded before and during surgery both with and without adrenaline in the intraocular infusion. Measurements were made before administration of drops (baseline), before anaesthesia (pre-anaes), and before surgery (pre-surg), then at 10,20, and 30 minutes during surgery. Data points are displaced slightly in time to facilitate comparison of standard error bars.

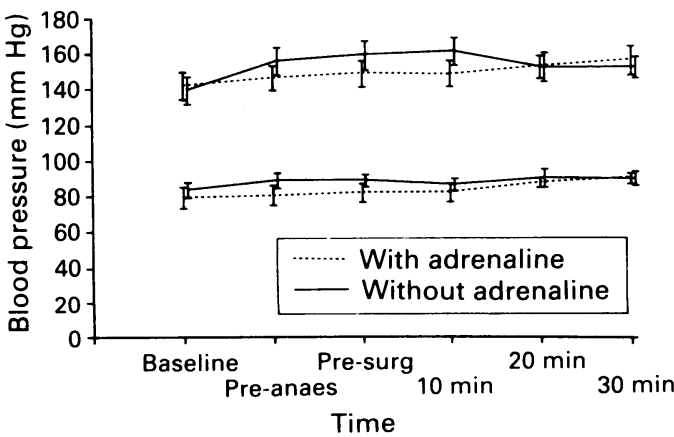

Figure 4 Systolic and diastolic blood pressure before and during surgery both with and without adrenaline in the intraocular infusion. Measurements were made before administration of drops (baseline), before anaesthesia (preanaes), and before surgery (pre-surg), then at 10, 20, and 30 minutes during surgery. Data points are displaced slightly in time to facilitate comparison of standard error bars. 
patient not receiving adrenaline, when the pupil was $8 \mathrm{~mm}$ in diameter.

The pulse rate (Fig 3 ) and blood pressure (Fig 4 ) in the 27 patients who had local anaesthesia showed no significant difference $(p>0.05)$ between the treatment groups. There was no increased incidence of ventricular dysrhythmias in the group receiving adrenaline.

\section{Discussion}

Cataract extraction is, in the majority of cases, a safe and effective procedure, but maintenance of mydriasis can contribute to the ease with which surgery can be performed. ${ }^{15} \mathrm{~A}$ small pupil during surgery may increase the risk of damage to the iris, incomplete clearance of soft lens matter, or more importantly, rupture of the posterior capsule. $^{2}$

To maintain mydriasis during surgery, some surgeons use adrenaline either in the intraocular irrigation fluid, or as a bolus injection into the anterior chamber. Studies of the effect of a bolus injection of adrenaline have shown that the pupil is significantly larger 1 minute after the injection compared with before,${ }^{6}$ but provide no data on whether this is maintained while surgery continues.

The present study demonstrates that adrenaline 1:1000000 in the intraocular infusion is of significant benefit in maintaining mydriasis during cataract surgery. It also reduces the number of eyes in which the pupil diameter becomes less than $5 \mathrm{~mm}$. One advantage of giving adrenaline in the irrigation fluid, as opposed to a bolus, is that it continues to enter the eye while the stimulus to miosis persists. As adrenaline is administered over a longer time period by infusion than by injection, a more dilute concentration can be used. In addition, the dose of adrenaline infused is dependent upon the duration of soft lens matter aspiration, and is therefore often related to the degree of miosis. If the pupil is large and aspiration is easy, less adrenaline will tend to be infused than in difficult cases with a small pupil. Being able to minimise the dose and concentration of adrenaline used helps to limit complications.

Early experience with intraocular adrenaline resulted in a few cases of severe corneal decompensation following use of the $1: 1000$ concentration, ${ }^{7}$ but not 1:5000. ${ }^{8}$ Corneal endothelial damage was shown to result from toxicity due to the sodium bisulphite preservative in the adrenaline preparation, rather than the adrenaline itself. ${ }^{8}$ It was also suggested that the osmolarity and $\mathrm{pH}$ of the solution may have been contributing factors, and showed that preparations with a low buffering capacity are less likely to cause corneal endothelial damage. ${ }^{9}$ The local safety of lower concentrations of adrenaline has since been demonstrated by histological studies of the endothelium in cats ${ }^{10}$ and rabbits, ${ }^{11}$ and by clinical examination in humans. ${ }^{112}$ Following a bolus injection in humans, 1:96000 adrenaline has been shown to be as effective as the 1:16000 concentration. ${ }^{6}$

Systemic absorption of adrenaline infused into the eye can potentially occur both via the vascular structures of the anterior segment, and via the nasolacrimal duct from overspill into the conjunctival sac. Following administration of retrobulbar anaesthetic containing adrenaline (0.8 $\mu \mathrm{g} / \mathrm{kg}$, average total dose $56 \mu \mathrm{g}$ ), a $190 \%$ increase in plasma adrenaline concentration has been demonstrated..$^{13}$ During intraocular irrigation with $1: 500000$ adrenaline $(1 \cdot 3 \mu \mathrm{g} / \mathrm{kg}$, average total dose $81.5 \mu \mathrm{g}$ ), plasma concentrations of adrenaline and noradrenaline did not differ significantly from those noted before induction of anaesthesia. ${ }^{14}$ In that study, heart rate increased following induction of anaesthesia, then remained unchanged thereafter, but there was no control group in which the normal response to anaesthesia and surgery was recorded for comparison. In a similar study on a mixed population of patients receiving local or general anaesthesia, there was no significant change in blood pressure or heart rate during the time of adrenaline administration. ${ }^{15}$ In the present study using 1:1000000 adrenaline (average total dose 40 $\mu \mathrm{g}$ ) in local anaesthetic cases, there was no significant difference in blood pressure or heart rate between the two treatment groups, and no significant variation from baseline. Neither was there an increased incidence of ventricular dysrhythmias in the group receiving adrenaline.

Adrenaline maintains mydriasis by a direct action on the dilator pupillae of the iris. An alternative approach is to inhibit the mechanism responsible for producing miosis, but this has not yet been precisely identified. ${ }^{16}$ At one time prostaglandins were considered likely candidates as they are present in the aqueous humour of normal eyes, ${ }^{17}$ and levels rise during trauma ${ }^{18}$ and cataract surgery. ${ }^{19}$ However, no prostaglandin has been shown to produce a dose dependent miotic response, ${ }^{16}$ and in fact most have been found to relax the rabbit iris sphincter. ${ }^{20}$

Recent years have seen the introduction of non-steroidal anti-inflammatory agents as an alternative or adjunct to the use of intraocular adrenaline. Some clinical studies have shown a mild but significant inhibition of miosis by indomethacin, ${ }^{21-24}$ flurbiprofen, ${ }^{23}{ }^{24}$ diclofenac, ${ }^{25}$ and suprofen, ${ }^{26}$ but others have shown virtually no effect. ${ }^{24}$ It appears that the extent of inhibition is correlated with the degree of miosis in the control group, and the amount of surgical trauma. ${ }^{16}$ These drugs inhibit the cyclooxygenase pathway responsible for the production of prostaglandins, thromboxane, and prostacyclins; and in higher doses, may also inhibit the lipoxygenase pathway. These agents should be used with caution as moderate inhibition of the cyclo-oxygenase pathway makes more arachidonic acid available to the lipoxygenase pathway, which increases the production of leukotrienes ${ }^{17}$ and stimulates intraocular inflammation. ${ }^{18}$ Corticosteroids inhibit phospholipase $\mathrm{A}_{2}$ thereby blocking arachidonic acid release at an even earlier stage in the pathway. They have a minimal effect in preventing miosis, and are not generally used for this purpose. ${ }^{21}$

Other agents which may have a role in surgically induced miosis include bradykinin, histamine, leukotrienes, substance $P$, vasoactive intestinal polypeptide, and other neuropeptides. Prostaglandins may act by modifying the release 
and activity of these other agents, rather than by having a direct effect. ${ }^{16}$

This study has shown that perioperative intraocular irrigation with adrenaline 1:1000000 has significant benefit in maintaining mydriasis during cataract surgery. The use of this concentration avoids the local and systemic complications associated with higher concentrations. Further research is needed to identify the mediators of surgically induced miosis, and it may then be possible to develop specific inhibitors which maintain mydriasis.

1 Williams HP. Manipulation of the iris in extracapsular len extraction. Trans Ophthalmol Soc UK 1985; 104: 553-7.

2 Goodman DF, Stark WJ, Gottsch JD. Complications of cataract extraction with intraocular lens implantation. Ophthalmol Surg 1989; 20: 132-40.

3 Lowenstein O, Levine AS. Pupillographic studies. V. Periodic sympathetic spasm and relaxation and role of sympathetic nervous system in pupillary innervation. Arch Ophthalmol 1944; $31: 74$.

4 Thompson HS. The pupil. In: Moses RA, Hart WM Jr, eds. Adler's physiology of the eye. St Louis: Mosby, 1989: 311-38. 5 Duffin RM, Canras CB, Gardner SK, Pettit TH Inhibitors of surgically-induced miosis. Ophthalmology 1982; 89: 966-79.

6 Duffin RM, Pettit TH, Straatsma BR. Maintenance of mydriasis with epinephrine during cataract surgery. Ophthalmic Surg 1983; 14: 41-5.

7 Dohlman CH, Hyndiuk RA. Subclinical and manifest corneal oedema after cataract extraction. Symposium on the cornea. Transactions of the New Orleans Academy of Ophthalmology. St Louis: Mosby, 1972: 221 .

8 Hull DS, Chemotti MT, Edelhauser HF, Van Horn DL, Hyndiuk RA. Effect of epinephrine on the corneal endoHyndiuk RA. Effect of epinephrine on the
thelium. Am $f$ Ophthalmol 1975; 79: 245-50.

9 Edelhauser HF, Hyndiuk RA, Zeeb A, Schultz R. Corneal oedema and the intraocular use of epinephrine. Am $\mathcal{F}$ Ophthalmol 1982; 93: 327-33.

10 Olson RJ, Kolodner H, Riddle P, Escapini H. Commonly used intraocular medications and the corneal endothelium. Arch Ophthalmol 1980; 98: 2224-6.

11 Adenis JP, Jabalot L, Leboutet MJ, Loubet R. Experimental study of the toxicity of adrenaline on the corneal endothelium. Application to extracapsular cataract surgery. Bull Mem Soc Fr Ophtalmol 1985; 96: 428-31.

12 Freeman JM, Gettelfinger TC. Maintaining pupillary dilatation during lens implant surgery. Am Intra-Ocular Implant Soc F 1981; 7: 172-3.
13 Donlon JV, Moss J. Plasma catecholamines during loca anaesthesia for cataract operations. Anaesthesiology 1979; 51 471-3.

14 Fell D, Watson AP, Hindocha N. Plasma concentrations of catecholamines following intraocular irrigation with adrenaline. Br $\mathcal{F}$ Anaesth 1989; 62: 573-5.

15 Fiore PM, Cinotti AA. Systemic effects of intraocular epinephrine

16 Camras CB, Miranda OB. The putative role of prostaglandins in surgical miosis. In: Bito LZ, Stjernschantz J, eds. Progress in surgical miosis. In: Bito LZ, Stjernschantz J, eds. Progress

17 Kulkarni PS, Srinivasan BD. Cyclooxygenase and lipoxygenase pathways in anterior uvea and conjunctiva. In: Bito LZ, Stjernschantz J, eds. Progress in clinical and biological research 1989; 321: 39-52.

18 Bhattacherjee $P$. The role of arachidonate metabolites in ocular inflammation. In: Bito LZ, Stjernschantz J, eds. Progress in clinical and biological research 1989; 321: 211-27.

19 Miyake K, Sugiyama S, Norimatsu I, Ozawa T. Prevention of cystoid macular edema after lens extraction by topical indomethacin. III. Radioimmunoassay measurements of prostaglandins in the aqueous during and after lens extracpion procedures. Graefes Arch Clin Exp Ophthalmol 1978; 209: 83-8.

20 Miranda OC, Bito LZ. The putative and demonstrated miotic effects of prostaglandins in mammals. In: Bito LZ Stiernschantz $\mathrm{J}$ eds. Progress in clinical and biological Stjernschantz J, eds. Progr

21 Dube P, Biosjoly HM, Bazin R, Chamberland G, Laughre PA, Dube I. Comparison of prednisolone acetate and indomethacin for mydriasis during cataract surgery. $\operatorname{Can} \mathcal{J}$ Ophthalmol 1990; 25: 243-8.

22 Keulen-De Vos HC, Van Rij G, Renardel De Lavelette JCG, ansen JTG. Effect of indomethacin in preventing surgically induced miosis. Brf Ophthalmol 1983; 67: 94-6.

23 Sachdev MS,Mehta MR, Dada VK, Jain AK, Garg SP, Gupta SK. Pupillary dilatation during cataract surgery - relative efficacy of indomethacin and flurbiprofen. Ophthalmic Surg 1990; 21: 557-9.

24 Psilas K, Kalogeropoulos C, Loucatzicos E, Asproudis I Petroutsos G. The effect of indomethacin, diclofenac and flurbiprofen on the maintenance of mydriasis during extracapsular cataract extraction. Doc Ophthalmol 1992; 81: 293-300.

25 Ertuck H, Ozcetin H, Cavci R. Diclofenac sodium for the prevention of surgically induced miosis. Eur $\mathcal{F}$ Implant Ref Surg 1991; 3: 55-7.

26 Stark WJ, Fagadau WR, Stewart RH, Crandall AS, deFalle JM, Reaves TA, et al. Reduction of pupillary constriction during cataract surgery using suprofen. Arch Ophthalmol $1986 ; 104: 364-6$.

27 Gimbel HV. The effect of treatment with topical nonsteroida anti-inflammatory drugs with and without intraoperative epinephrine on the maintenence of mydriasis during cataract surgery. Ophthalmology 1989; 96: 858-8. 